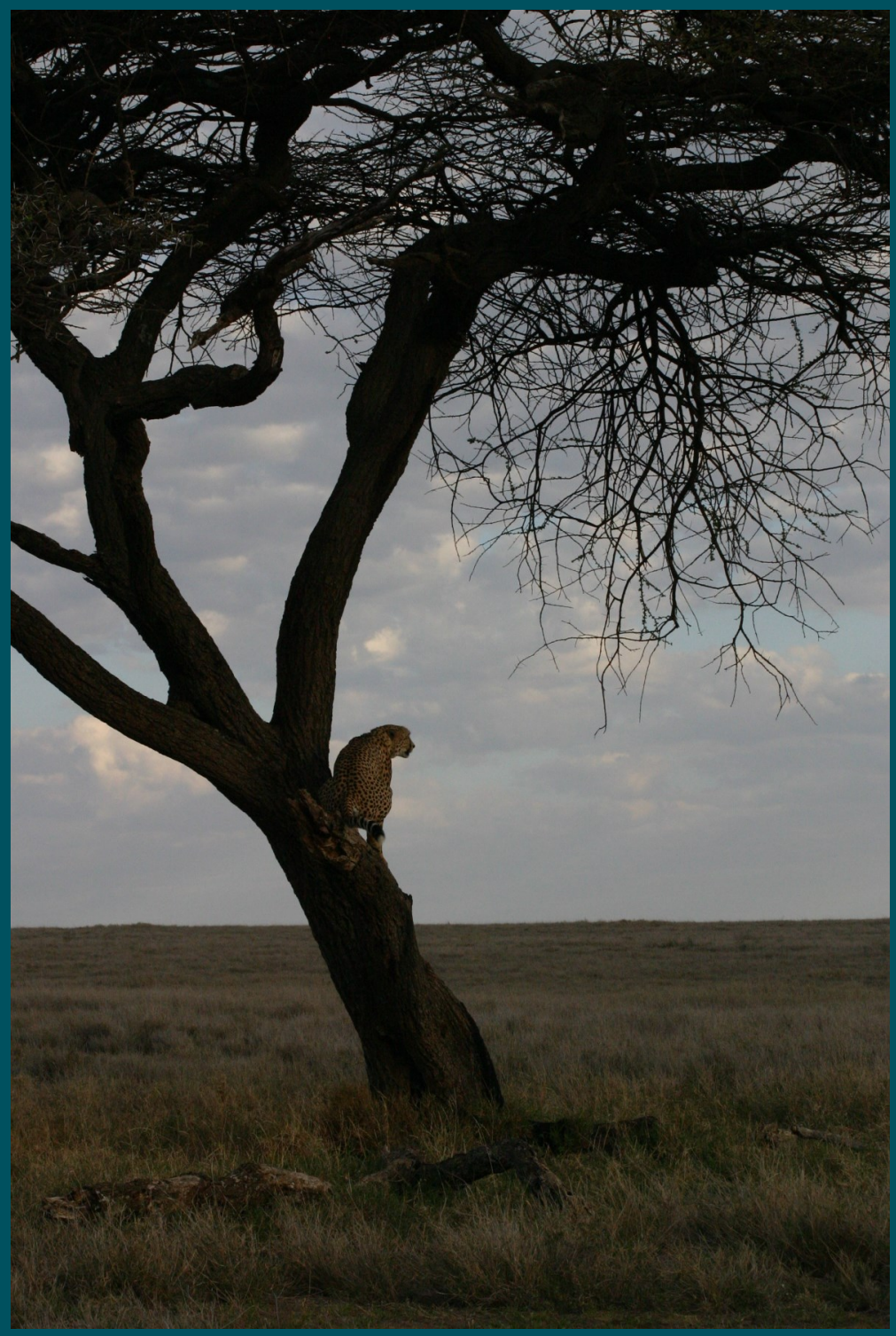

\title{
Pause and Reflect
}

A Simple Tool for Teams to Learn and Agree How to Do Things Better

David Wilkie and Heidi Kretser Rights + Communities Program

December 1, 2021 


\section{What is Pause and Reflect?}

A Pause and Reflect Session is a simple process for teams to meet regularly or in response to a crisis to ask themselves how things are going, what is going as planned, are they making progress, is there anything they need to change to move ahead better or faster, and what have they learned that might be worth sharing with others.

It is one of the best ways for teams to learn together, to adapt and improve their work as they learn new things, and to share what they are learning with others.

\section{Who uses Pause and Reflect?}

Pause and Reflect Sessions are used by many different types of organizations. The military all over the world uses Pause and Reflect sessions after every patrol or deployment - they call them After Action Reviews. Hospital emergency room staff use them at the end of each day to talk about what treatments worked best and what treatments could be improved.

Maybe this will be surprising but Football coaches use Pause and Reflect Sessions every game at half-time to ask the team what is working, and what do they need to change and win the game.

So you can see that many different teams use Pause and Reflect to talk about how their work is going and what if

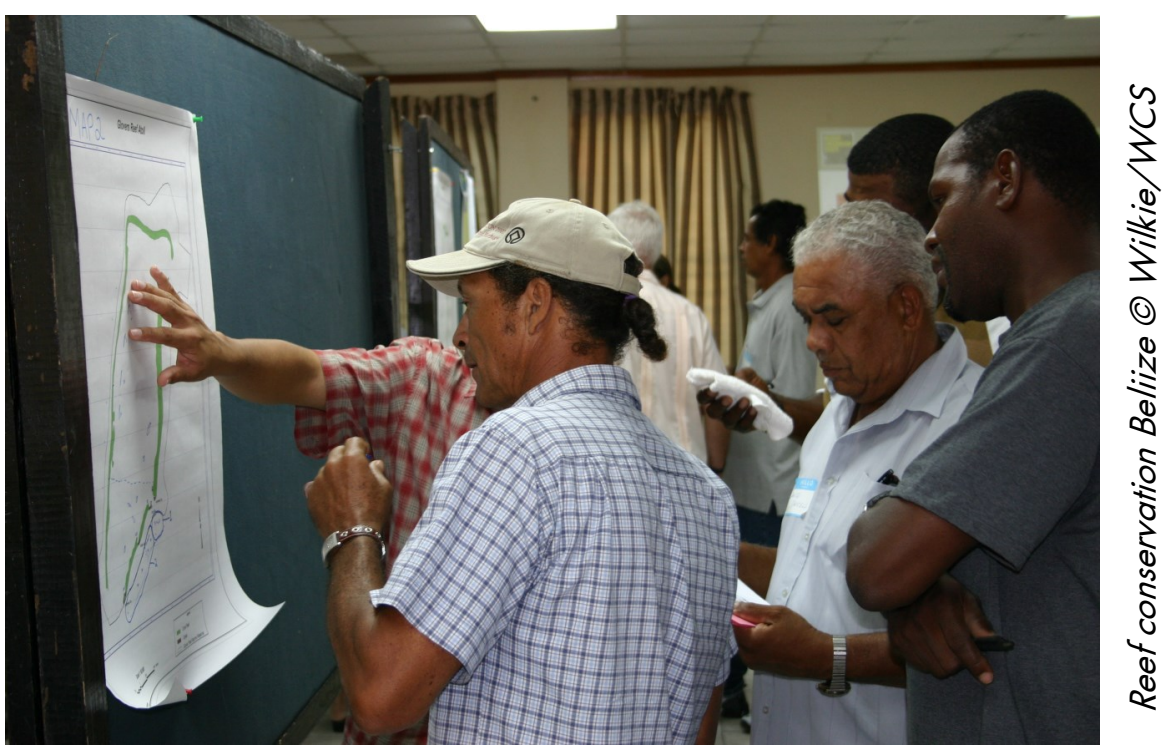




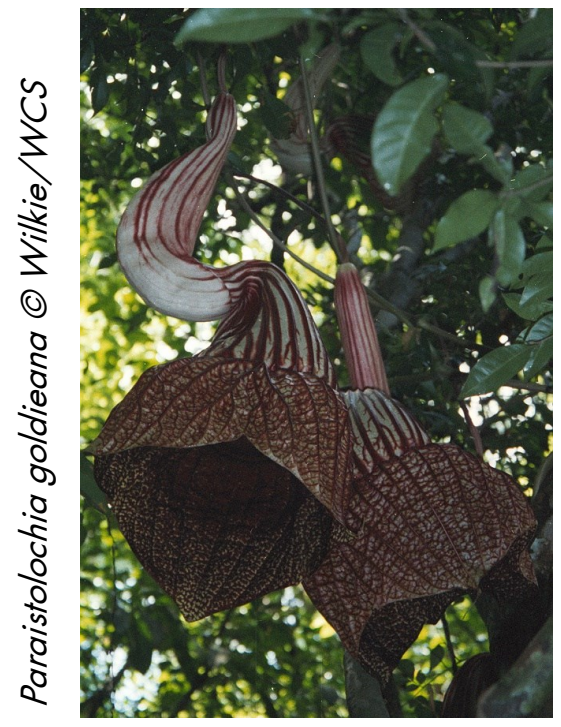

anything they might need to change to work better, smarter and more effectively.

Conservation organizations have only recently started to use Pause and Reflect Sessions and WCS is one of the first to start using this team building process to increase our conservation effectiveness.

\section{When should we use Pause and Reflect?}

Team learning through Pause and Reflect discussions can occur as a regularly scheduled part of the team's work, to discuss day to day successes as well as activities that may not be going as planned. Special sessions of Pause and Reflect can be implemented after a crisis has happened or failure has been observed.

Conducting regularly scheduled team Pause and Reflect sessions is an essential way for teams to learn the process of frank open discussion without "blame gaming." This arms them with the experience they need to conduct a Pause and Reflect session when something bad or unexpected has occurred that needs to be understood and addressed.

An important take home message is, that teams need to practice Pause and Reflect while things are going relatively well, before a crisis happens and they really need to Pause and Reflect.

\section{What do we want to achieve with each Pause and Reflect session?}

At the end of each Pause and Reflect session we want to accomplish two things:

1. We want to have had an honest and open discussion where everyone in the team felt comfortable sharing their thoughts and opinions, and

2. We want to have a clear set of action steps that we all agree will help us all work better as a team moving forward 


\section{What process will we follow for each Pause and Reflect session?}

Anyone in the team can facilitate the Pause and Reflect session. The most important role of the facilitator is to encourage everyone to contribute to the discussion, to layout the topics of discussion (see below) at the beginning of the session (even better to share these when scheduling the session), and keep an eye on the clock to make sure that all topics on the list are discussed within the allotted time.

- What did we hope to achieve?

- What went well? Why?

- What didn't go as well as we hoped? Why?

- What have we learned?

- What can we do better?

- Are there any things we should stop doing or do differently?

- What action steps are we going to take as a team moving forward?

It is important to record highlights of the discussion and the action steps the team agrees to take based on what they learned during the session. The facilitator can also be the note taker or can ask another team member to serve that role. Notes are best taken using a real or virtual (projected) flip chart or whiteboard. After the session the facilitator should communicate with the team, thanking them for their participation, and sharing the notes and action steps. It is also useful to archive notes from all Pause and Reflect sessions in a shared Google/One Drive folder or on a Slack channel 


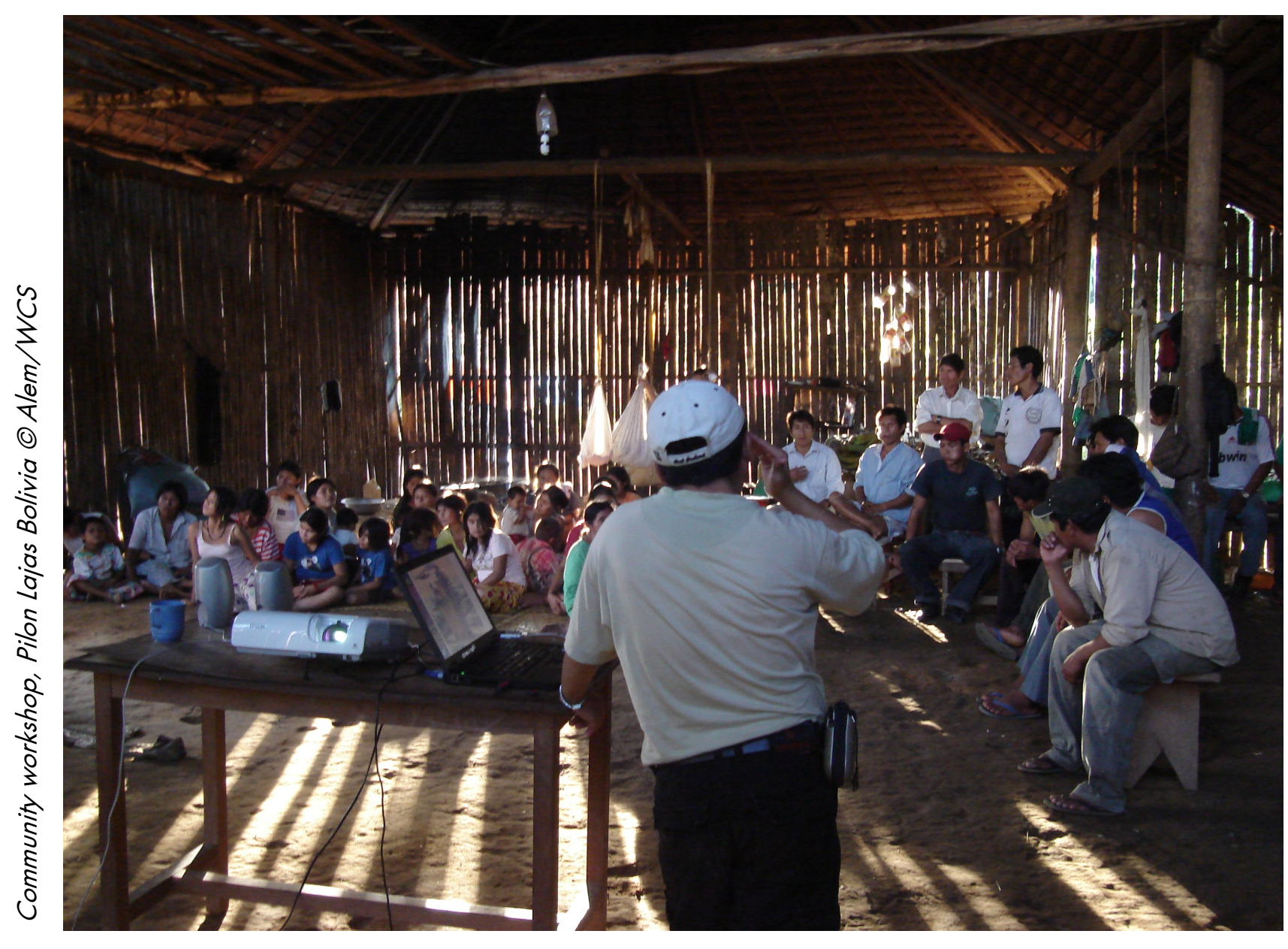

\section{Advice to the team}

\section{from lain Dickson, BirdLife}

- Don't interrupt teammates

- Listen without criticism or judgment to what team members have to say

- Focus on underlying reasons/root causes

- Explain what constructive action should be taken

- Pause and Reflect sessions will NOT be used to assess individual performance but to drive collective improvements in practice 


\section{Wildlife Conservation Society Global Conservation Program 2300 Southern Boulevard Bronx, NY 10460 Tel: $718-220-5100$ WWW. WCS.org}

\section{Wildife Conservation Society}

